\title{
Assessment of cardiac apex impulse using fiberoptics
}

\author{
James S. Cole and Robert D. Conn ${ }^{1}$ \\ From the Division of Cardiology, Department of Medicine and \\ Center for Bioengineering, University of Washington, \\ Seattle, Washington, U.S.A.
}

The fiberoptic transducer described in this report meets the necessary criteria for accurately recording the absolute movement of the cardiac apex impulse, and provides some advantages over the instruments in current use. It records total vertical displacement of the impulse, is sensitive to ultralow frequency vibrations, does not contact the chest wall, and is easily adapted to standard office or laboratory recording equipment, thereby making it practical for routine clinical instruction and investigations. It was found to reproduce reliable tracings in patients with left ventricular hypertrophy, and repeatedly recorded an impulse with 'normal' characteristics in patients without left ventricular hypertrophy or dilatation, and should therefore reflect accurately what the clinician feels and sees at the bedside. Fiberoptics prove to be of value for the continued investigation of praecordial impulses.

\begin{abstract}
'It has been a constant endeavor on my part to recognise the different conditions which these instruments have revealed by employing the ordinary bedside methods of examination.'
\end{abstract}

(Mackenzie, 1916)

The cardiac apex impulse provides invaluable information regarding structural and functional abnormalities of the left ventricle. Various instruments have been devised to record the characteristics of the apex impulse, and from these recordings assumptions have been made regarding the clinical implications of the impulse. Unfortunately, the mechanical properties of the instruments in common use are dissimilar, and a representative wave form for the apex impulse has not been established.

Two general types of instruments have been used for recording praecordial motion. The commonly used apex cardiograph records the movement of the apex impulse relative to surrounding praecordial movement and produces a wave form that differs from that which the clinician observes and palpates. The second group of instruments, including the kinetocardiograph and the impulse cardiograph, records absolute impulse displacement

Received 17 August 1970.

${ }^{1}$ Address communications to Dr. Robert D. Conn, Physician-in-Chief, Harborview Medical Center (King County Hospital), 325 Ninth Avenue, Seattle, Washington 98104, U.S.A. and more accurately represents clinical observations. Each of these instruments, however, has practical and theoretical disadvantages that have limited their widespread use.

The ideal instrument should record absolute praecordial displacement, impart minimal impedance to movement as it contacts the chest wall, and the transducer should be accurate over the very low vibratory frequency range $(0-15 \mathrm{~Hz})^{1}$. The entire system must also be practical for routine clinical use.

The purpose of this report is (I) to describe a new fiberoptic transducer that uses reflected light for recording praecordial motion, (2) to describe the contour of the impulse recorded with this transducer, (3) to contrast it with the standard apex cardiogram, and (4) to discuss the advantages of this system over other currently available systems.

\section{Description of equipment}

A fiberoptic transducer (KD-38), manufactured by Mechanical Technology Incorporated, Instrument Division, Latham, New York, was evaluated. The probe is $0.109^{\prime \prime}$ in diameter and contains 600 randomly arranged strands of glass fiberoptics (Fig. I). Three hundred strands transmit light which is emitted in a narrow conical distribution. The light is reflected from an area of approximately $0.1 \mathrm{~cm}^{2}$ on the test surface, and a portion of the reflected light is received by the remaining

1 (I $\mathbf{H z}=$ I cycle per second). 


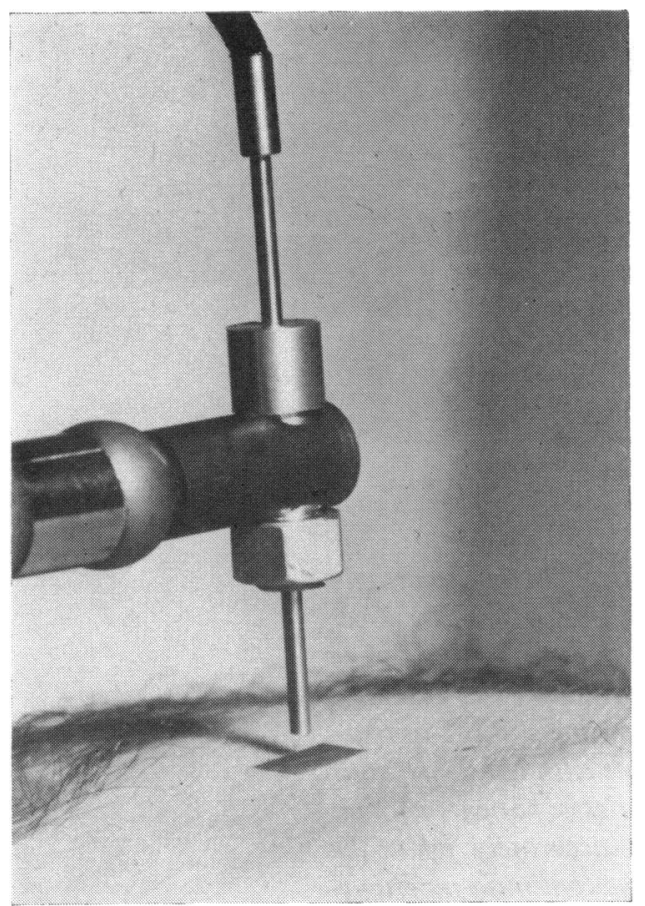

FIG. I Fiberoptic probe positioned above cardiac apex impulse. Aluminium foil used to reflect light.

300 strands. The amount of light received is inversely proportional to the distance between the probe and reflecting surface (gap). The received light is converted into an analogue output signal and either read directly from the integral meter on the unit or displayed on a stylus writer or oscilloscope. A Hewlett-Packard ro56A Heart Sound Amplifier and 500A Viso-Cardiette Electrocardiogram were used to record the signals from the fiberoptic transducer. The signal from the transducer was put through the electrocardiogram channel of the heart sound amplifier and recorded on the $500 \mathrm{~A}$ Viso-Cardiette electrocardiogram recorder. A switch allows selective recording of either the signal from the fiberoptic transducer or the phonocardiogram, or superimposition of the phonocardiogram on the signal from the fiberoptic transducer for simultaneous recording. This is, in essence, a dual channel tracing, using medical office equipment (Fig. 2). The tracing may also be recorded on a multichannel recording unit since the output of the transducer is suitable for standard electrocardiogram amplifiers.

Specifications of fiberoptic transducer Fig. 3a shows the output/gap (distance from probe to test surface) curve relating the output of the transducer to light of constant intensity reflected from a source at variable distances. The response is linear to within 2 per cent on the 'down slope' of the curve when the gap is between
2 and $5 \mathrm{~mm}$. This represents the gap at which clinical recordings are made. The output frequency response is flat from DC to $15 \mathrm{KC}$. The output impedance is $4 \mathrm{~K} \mathrm{Ohm}$, and the output level is I volt DC at full-scale meter deflection.

The fiberoptic transducer and recorder were initially evaluated using a mechanical shaker as the vibrating surface. (Ling shaker, A-286; power amplifier, RP $3 / 5$, and servocycling oscillator SCO 100 with frequency of 5 to $5,000 \mathrm{~Hz}$, and stroke amplitude of $I$ in peak-to-peak maximum.) A linear output was obtained when the baseline gap was set at $4 \mathrm{~mm}$, the frequency at $7.5 \mathrm{~Hz}$, and the amplitude of the shaker increased from 0.3 to $2.5 \mathrm{~mm}$ (Fig. 3b). The output was also linear when the amplitude of the shaker was held constant (I.2 mm peak-to-peak) and the frequency of the shaker increased from 5 to $15 \mathrm{~Hz}$ (Fig. 3c), and when the frequency and amplitude of the shaker were held constant and the gap varied from 2 to $5 \mathrm{~mm}$ (Fig. 3d). There was a rapid decline in output below $2 \mathrm{~mm}$ and above $5 \mathrm{~mm}$. There was no significant distortion of the signal until the angle of the probe was more than Io degrees from a line perpendicular to the reflecting surface.

\section{Clinical methods}

After calibration, the instrument was used to record praecordial movement in 25 normal people and 50 patients with congenital or valvular heart disease. A piece of aluminium foil $(\mathrm{I} \times \mathrm{I} \mathrm{cm})$ was placed over the area of the apex impulse where maximal praecordial activity was observed. The probe was fixed above the praecordium using a

FIG. 2 Fiberoptic recording system using electrocardiograph and $a$ stand with $a$ flexible multijointed arm for positioning probe above apex impulse.

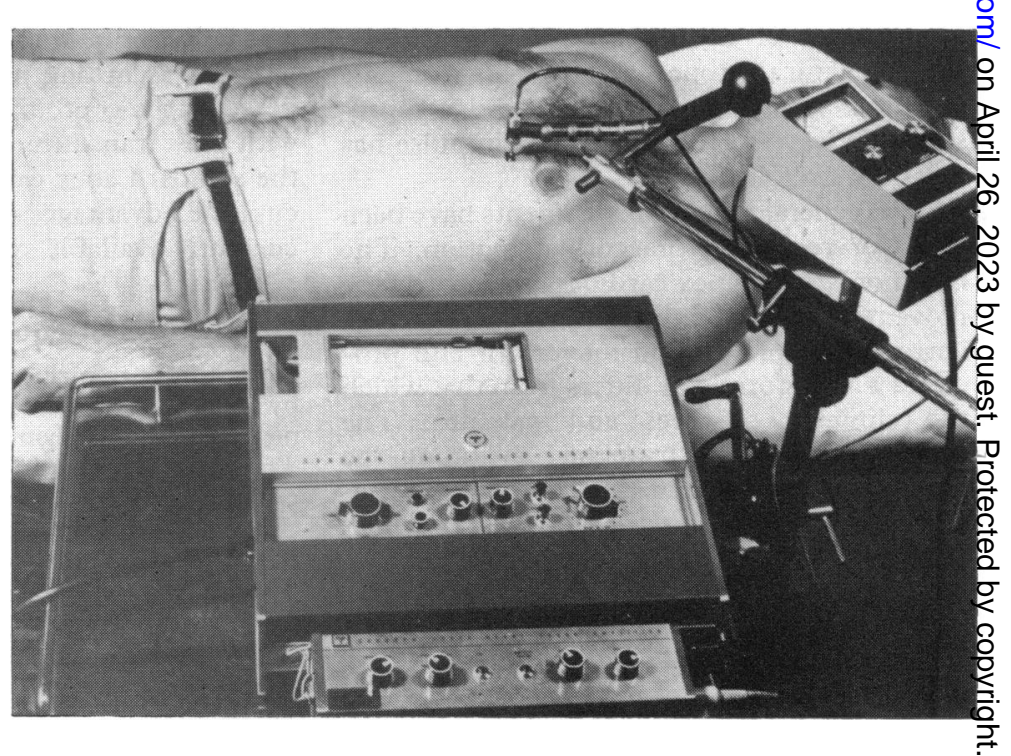


flexible, multijointed metal arm which permitted easy positioning of the probe tip (Fig. 2). Recordings were taken in the supine position during suspended expiration. The gap between probe and reflector tape was approximately $3 \mathrm{~mm}$, and the baseline output was adjusted to 50 per cent of maximum, to obtain a linear output.

Twenty-five normal subjects and 50 patients were studied. The normal subjects were selected from among patients, medical students, and hospital employees with a negative cardiac history, normal chest $x$-ray, and electrocardiogram, and normal cardiac examination. The 50 patients were selected from patients undergoing cardiac catheterization, including quantitative left ventricular angiography. Left ventricular mass was determined by the method of Rackley (Rackley et al., 1964). Using this method, the upper limit of normal for left ventricular mass is $124 \mathrm{~g} / \mathrm{m}^{2}$ of body surface area (Kennedy et al., 1966).

\section{Results}

A recording of ultralow frequency praecordial movement was easily obtained. An identical pattern was obtained on Io to I5 successive beats, and a similar pattern could be obtained from a specific patient on repeated tracings. Praecordial movement and phonocardiograms were recorded without difficulty either separately or simultaneously in normal subjects and patients with heart disease.

Fig. 4 shows the simultaneous recording of the phonocardiogram and fiberoptic impulse cardiogram in a normal subject and in a patient with aortic stenosis and left ventricular hypertrophy. The normal impulse tracing on the left is recorded above the baseline in early systole but returns to and falls below the baseline in the last one-third of systole. This type of recording was obtained in all 25 normal control subjects. The abnormal tracing on the right also is recorded above the baseline in early systole, but rather than returning to the baseline in late systole, remains positive throughout systole. This type of prolonged impulse tracing was obtained in 28 of 32 patients with increased left ventricular mass as determined angiographically $(\mathrm{P}<0.00 \mathrm{I})$. In the majority of these cases the positive impulse extended into early diastole, producing a recorded impulse that exceeded the duration of mechanical systole. The recorded impulse returned to the baseline in the last one-third of systole in only 4 of the 32 patients, with angiographically documented left ventricular hypertrophy. In contrast, 18 catheterized patients had normal left ventricular mass (less than $124 \mathrm{~g} / \mathrm{m}^{2}$ body surface area). Fourteen of these patients had a recorded impulse that returned to the base-
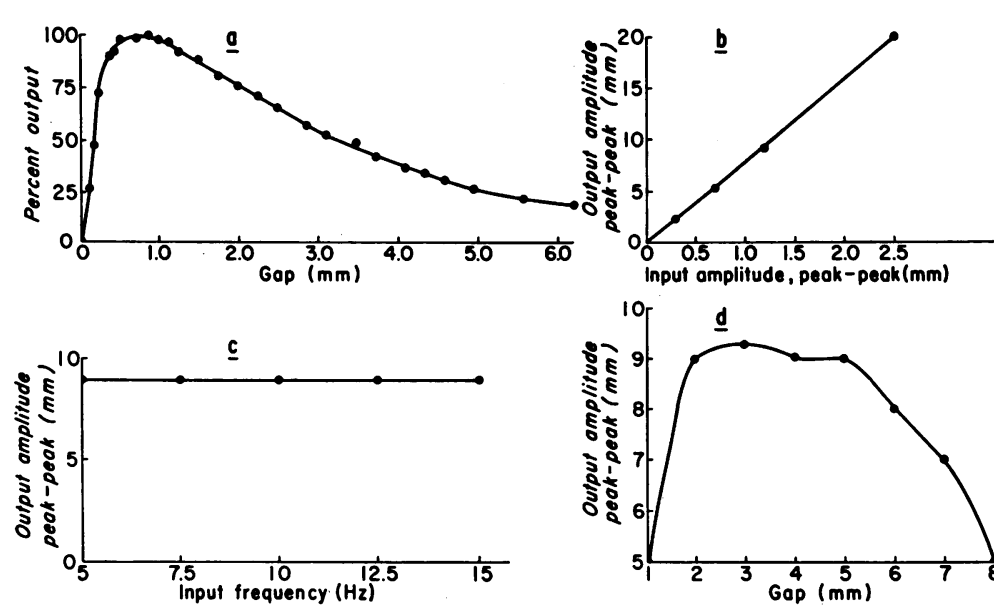

FIG. 3 Calibration. (a) Relation of the percentage output of the transducer to the distance from the probe to the reflecting surface (gap). (b) Relation of amplitude of vibrating test surface (input amplitude) to amplitude recorded on electrocardiograph recorder (output amplitude). (c) Demonstration of constancy of output of fiberoptic transducer over the low frequency range. (d) Demonstration of relatively linear output from fiberoptic transducer for a gap between 2 and $5 \mathrm{~mm}$.

line by the last one-third of systole, while only 4 had a recorded impulse equal to or exceeding the duration of systole $(\mathrm{P}<0.00 \mathrm{I})$.

\section{Discussion}

Transducers currently used to record praecordial motion fall essentially into two groups. One type, represented by the standard apex cardiograph (Benchimol and Dimond, 1963; Tavel, I967; Tafur, Cohen, and Levine, 1964a), consists of a circular funnel connected by a short rubber tube to an active sensing element. The latter is either a piezoelectric crystal or a strain gauge. Vibrations from the skin surface under the funnel are transmitted through the air in the funnel and rubber tube to the sensing element where a signal is produced. The output from the sensing element is transmitted to an amplifier and the appropriate recording instrument.

The essential feature of the second type of transducer is its point of fixation above the chest surface. Both the kinetocardiograph (Eddleman et al., 1953) and the impulse cardiograph (Beilin and Mounsey, 1962) consist of a thin rod connected to a sensing element. The latter is fixed in space above the chest surface, while the tip of the rod is 
applied to the skin surface. The praecordial impulse moves the rod with reference to the fixed sensing element, and a signal proportional to displacement of the rod is produced. The sensing element in the kinetocardiograph is a collapsible air-filled bellows and piezoelectric crystal; and in the impulse cardiograph, a photoelectric cell.

The standard apex cardiograph has several disadvantages for recording the systolic portion of the apex impulse. The funnel as used encloses an area of $18 \mathrm{~cm}^{2}$. The signal produced is directly related to the volume of air compressed by movement of the enclosed skin surface. The total air displacement and therefore the signal produced is the average movement of all points enclosed by the funnel and not the movement of a specific localized point.

Secondly, since the funnel of the apex cardiograph is fixed to the chest surface, the movement recorded represents the differential

FIG. 4 Matched clinical recordings. (a) Phonocardiogram. (b) Apex impulse recorded with fiberoptic transducer. (c) Simultaneous recordings of phonocardiogram and apex impulse. Impulse recordings on the left represent a normal impulse that is positive in early systole and retracted in late systole. Recordings on the right represent the sustained (holosystolic) positive impulse observed in a patient with left ventricular hypertrophy. PCG, phonocardiogram. $S_{1}$, first heart sound. $S_{2}$, second heart sound.

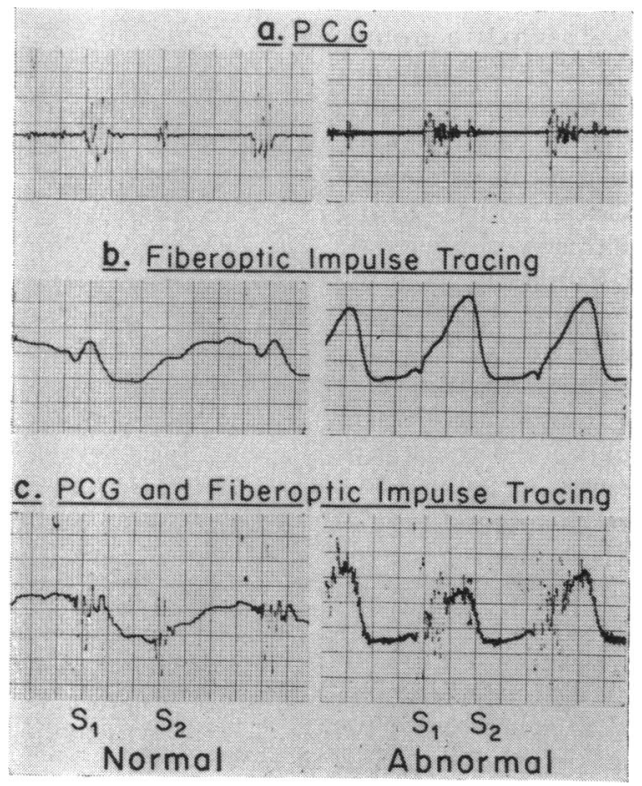

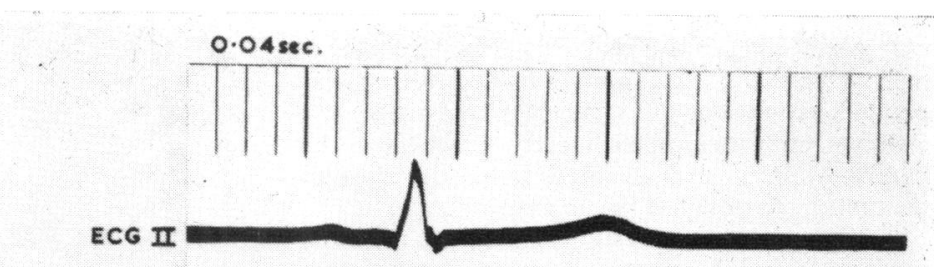
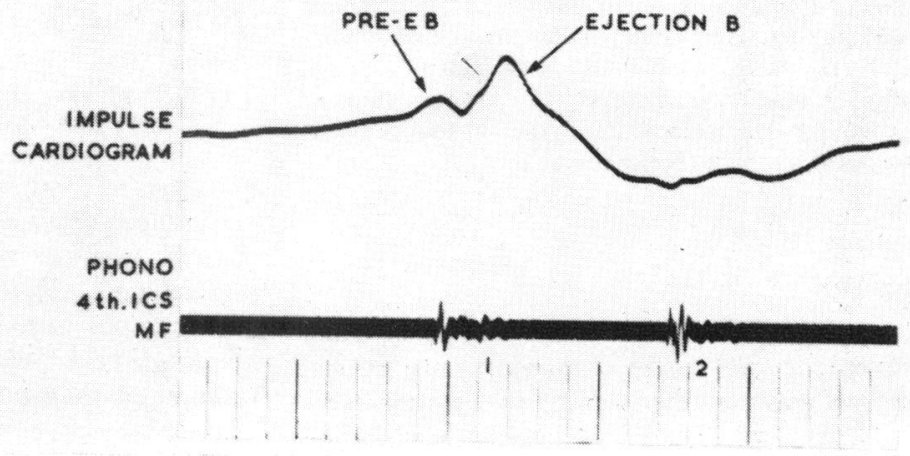

FIG. 5 Normal apex impulse recorded with the impulse cardiograph. Note similarity to recordings from the fiberoptic transducer.

(Reproduced from Toutouzas and Shillingford, 1969, British Heart Journal, 31, 97, Fig. I, page 99, by permission of the authors and the publisher.)

movement between the area under the funnel and the surrounding chest surface, rather than the absolute vertical movement of the area under the funnel.

Several other factors influence the type of tracing obtained with the apex cardiograph. Bancroft and Eddleman (1967) demonstrated a significant phase shift and variable frequency response. The amount of pressure used in applying the funnel also significantly alters the output (Mounsey, 1967; Beilin and Mounsey, 1962). In addition, it is customary to record the apex cardiogram with the patient in the left lateral decubitus position. This position may distort the systolic characteristics of the impulse, since it brings the cardiac apex in constant apposition with the chest wall, producing a sustained or holosystolic impulse in many patients, including those with a normal left ventricle. In this position it may also be difficult to obtain recordings with reproducible wave forms. For these reasons the apex cardiograph has not been of uniform assistance in recording the systolic events of 
left ventricular contraction, though it has provided major usefulness in the evaluation of diastolic movements.

The apex cardiograph, kinetocardiograph, and impulse cardiograph all contact the skin surface and may impose an undesirable preload on the surface with subsequent distortion or damping of the signal.

The kinetocardiograph also has features which result in distortion of the signal. Mounsey (1967) has pointed out that the airfilled bellows introduces a certain degree of adiabatic effect which tends to accentuate the peaks and troughs of the tracing. The stiffness of the bellows may also introduce additional inertia into the system.

The impulse cardiogram has not gained universal acceptance for the recording of apex impulse, but of the instruments in use it probably records most accurately the impulse as it is felt by the clinician. Though the impulse cardiogram touches the chest wall and imposes some preload on the area of the impulse, the magnitude may not be great ( 100 to $200 \mathrm{~g}$ ) (Beilin and Mounsey, 1962). The wave form of the apex impulse recorded by the fiberoptic transducer closely resembles that recorded by the impulse cardiogram (Fig. 5).

The major advantage of the fiberoptic transducer over the standard apex cardiogram is found in recording and interpreting the systolic events of the apex impulse. Clinicians associate an impulse of short duration with a normal left ventricle, while a sustained impulse is found in diseases producing left ventricular hypertrophy. Studies to date have shown that the apex impulse may result from the displacement of the praecordium by the cardiac apex or the left ventricular portions of the interventricular septum (Deliyannis et al., 1964). During early systole the apex (or apical septum) rotates anteriorly and results in a praecordial impulse. As systole progresses, the ventricular chamber decreases in size and pulls away from the chest wall. The normal left ventricle therefore produces an impulse that is positive during the first one-half to two-thirds of systole, but which is usually negative or retracted during the last third of systole (Deliyannis et al., 1964; Beilin and Mounsey, 1962; Cole and Conn, 1969), while the hypertrophied left ventricle produces an impulse that is 'sustained' or holosystolic. The primary difference from the normal impulse is that the abnormal impulse is still deflected outward during the last third of systole.

The fiberoptic transducer described in this report recorded an impulse of short duration
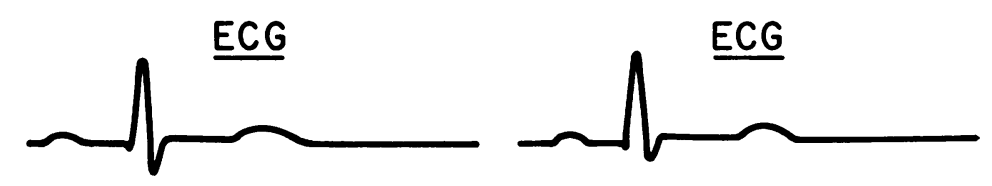

Fiberoptic Impulse Tracing
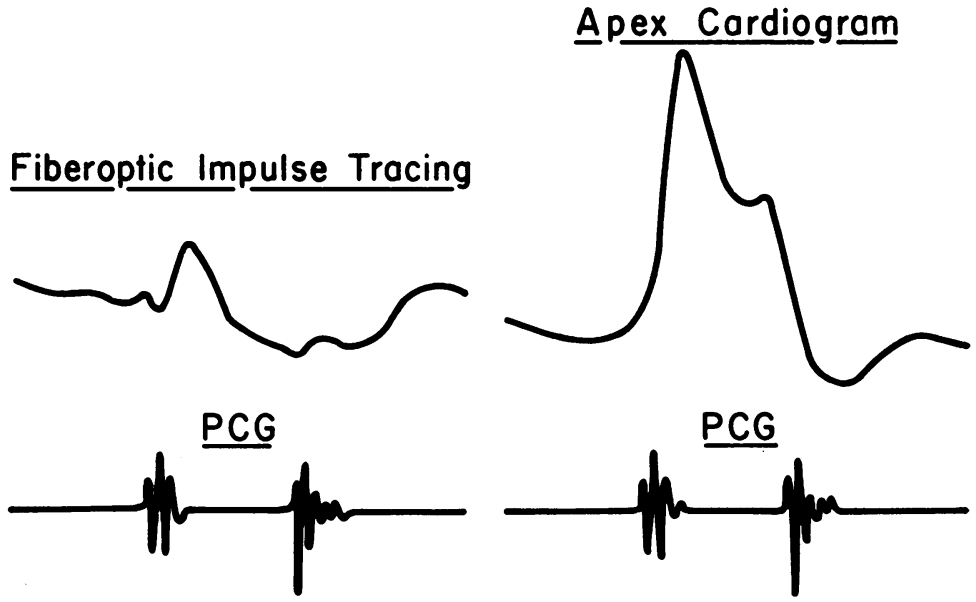

FIG. 6 Apex impulse recorded in the same patient using the fiberoptic transducer (left), and the standard apex cardiogram (right). Note that the fiberoptic transducer records a positive impulse of short duration, and the apex cardiogram records a prolonged (holosystolic) impulse (see text).

in the majority of patients with a normal left ventricle and a prolonged impulse in 28 of 32 patients with ventricular hypertrophy. In contrast, the standard apex cardiogram often records an impulse that is positive throughout systole even in normal subjects. Studies using the apex cardiogram (Tafur, Cohen, and Levine, 1964b; Sutton and Craige, 1967; Benchimol, Legler, and Dimond, 1963; Tavel, 1967) show that the impulse recorded by this technique does not return to the baseline until after the second heart sound has occurred in both normal subjects and patients with left ventricular hypertrophy. Fig. 6 compares the apex impulse recorded sequentially by the fiberoptic transducer and a standard apex cardiograph in a patient with a normal left ventricle. The fiberoptic impulse tracing is positive during only the first one-half of systole while the impulse recorded in the same position by the standard apex cardiograph is positive through systole. This suggests that the impulse recordings from the fiberoptic transducer predict left ventricular hypertrophy with more accuracy than the standard apex cardiogram and also correlate more closely with the type of impulse palpated by the clinician. 


\section{References}

Bancroft, W. H., Jr., and Eddleman, E. E. (1967). Methods and physical characteristics of the kinetocardiographic and apexcardiographic systems for recording low-frequency precordial motion. American Heart fournal, 73, 756.

Beilin, L., and Mounsey, P. (I962). The left ventricular impulse in hypertensive heart disease. British Heart fournal, 24, 409.

Benchimol, A., and Dimond, E. G. (1963). The apexcardiogram in normal older subjects and in patients with arteriosclerotic heart disease. Effect of exercise on the ' $a$ ' wave. American Heart fournal, 65, 789.

Benchimol, A., Legler, J. F., and Dimond, E. G. (1963). The carotid tracing and apexcardiogram in subaortic stenosis and idiopathic myocardial hypertrophy. American fournal of Cardiology, II, 427.

Cole, J. S., and Conn, R. D. (1969). Cardiac apex impulse: clinical and angiographic correlations (Abstract). Annals of Internal Medicine, 70, 1075.

Deliyannis, A. A., Gillam, P. M. S., Mounsey, J. P. D., and Steiner, R. E. (1964). The cardiac impulse and the motion of the heart. British Heart fournal, 26, 396.
Eddleman, E. E., Jr., Willis, K., Reeves, T. J., and Harrison, T. R. (1953). The kinetocardiogram. I. Method of recording precordial movements. Circulation, 8, 269.

Kennedy, J. W., Baxley, W. A., Figley, M. D., Dodge, H. T., and Blackmon, J. R. (1966). Quantitative angiocardiography. I. The normal left ventricle in man. Circulation, 34, 272.

Mackenzie, J. (1916). Principles of Diagnosis and Treatment in Heart Affections. Henry Frowde; Hodder and Stoughton, London.

Mounsey, J. P. D. (1967). Inspection and palpation of the cardiac impulse. Progress in Cardiovascular Disease, 10, 187.

Rackley, C. E., Dodge, H. T., Coble, Y. D., and Hay, R. E. (1964). A method for determining left ventricular mass in man. Circulation, 29, 666.

Sutton, G. C., and Craige, E. (1967). Quantitation of precordial movement. I. Normal subjects. Circulation, 35, 476 .

Tafur, E., Cohen, L. S., and Levine, H. D. (I964a). The normal apex cardiogram. Circulation, 30, 38I.

Tafur, E., Cohen, L. S., and Levine, H. D. (1964b). The apex cardiogram in left ventricular outflow tract obstruction. Circulation, 30, 392.

Tavel, M. E. (1967). Clinical Phonocardiography and External Pulse Recording. Year Book Medical Publishers, Chicago. 